\title{
UNIRATIONALITY OF CUBIC HYPERSURFACES
}

\author{
JÁNOS KOLLÁR
}

A remarkable result of [Segre43] says that a smooth cubic surface over $\mathbb{Q}$ is unirational iff it has a rational point. [Manin72, II.2] observed that similar arguments work for higher dimensional cubic hypersurfaces satisfying a certain genericity assumption over any infinite field. CT-S-SD87, 2.3.1] extended the result of Segre to any normal cubic hypersurface (other than cones) over a field of characteristic zero. It is also clear that the result should hold for all sufficiently large finite fields, though the details were not worked out in general. Manin72, IV.8] settles the cubic surface case for finite fields with at least 34 elements. The aim of this note is to observe that a variant of the Segre-Manin method works for all fields and for all cubics:

Theorem 1. Let $k$ be a field and $X \subset \mathbb{P}^{n+1}$ a smooth cubic hypersurface of dimension $n \geq 2$ over $k$. Then the following are equivalent:

1. $X$ is unirational (over $k$ ).

2. $X$ has a k-point.

Similar results hold for singular cubic hypersurfaces, with a few exceptions.

Theorem 2. Let $k$ be a perfect field and $X \subset \mathbb{P}^{n+1}$ an irreducible cubic hypersurface of dimension $n \geq 2$ over $k$ which is not a cone over an $(n-1)$-dimensional cubic. Then the following are equivalent:

1. $X$ is unirational (over $k$ ).

2. X has a k-point.

3. $X$ has a smooth $k$-point.

3 (Nonperfect fields). Over nonperfect fields of characteristic 3, there are nonsingular cubic hypersurfaces of arbitrary dimension which are not unirational but do have a $k$ point (17). I have not been able to find examples in characteristic 2 .

Question 4. Unirationality of varieties is very poorly understood in general and there are very basic open questions. We do not even have a list of unirational surfaces and very few examples are known in higher dimensions. For instance, let $X$ be a smooth projective variety over 
$k$ such that $X$ is unirational over $\bar{k}$. Assume for simplicity that $k$ is infinite and consider the following properties:

1. $X$ is unirational (over $k$ ).

2. $X(k)$ is dense in $X$.

3. $X$ has a $k$-point.

It is clear that each property implies the next. They are equivalent for cubic hypersurfaces by (11). It is extremely unlikely that they are always equivalent, but no counter examples are known.

5 (Proof of (2.1) $\Rightarrow(2 \cdot 2) \Rightarrow(2 \cdot 3)$ ).

(2.1) $\Rightarrow(2.2)$ is clear for infinite fields. For finite fields it follows from [Nishimura55].

Assume (2.2) and let $x \in X$ be a $k$-point. We are done if $X$ is smooth at $x$. Otherwise $x$ is a double point and we can choose affine coordinates such that $x=(0, \ldots, 0)$ and $X$ is given by an equation $q\left(x_{1}, \ldots, x_{n}\right)+c\left(x_{1}, \ldots, x_{n}\right)=0$ where $q$ is quadratic and $c$ is cubic. Assume that there is a point $\left(p_{1}, \ldots, p_{n}\right) \in k^{n}$ such that $q\left(p_{1}, \ldots, p_{n}\right) \neq$ 0 . Then the line connecting the origin and $\left(p_{1}, \ldots, p_{n}\right)$ intersects $X$ in a single point outside the origin and this is a smooth $k$-point of $X$. Thus we are done unless $q$ vanishes everywhere on $k^{n}$.

However, if a homogeneous polynomial $f$ of degree $d$ vanishes on $k^{n}$ and $|k| \geq d$ then $f$ is identically zero.

The intersting part is to show unirationality starting with a smooth $k$-point. The construction is presented in 3 stages, successive version working in greater and greater generality. At least in retrospect, all of this is only a slight modification of the works of Segre.

6 (First unirationality construction).

Let $X \subset \mathbb{P}^{n+1}$ be a cubic and $p \in X$ a point. Let $C_{p}$ denote the intersection of $X$ with the tangent plane at $p$. We expect that usually $C_{p}$ is an irreducibe cubic with a double point at $p$. If this is indeed the case then the inverse of the projection from $p$ gives a birational map $\pi_{p}: \mathbb{P}^{n-1} \rightarrow C_{p}$. If $p \in X(k)$ then $C_{p}$ is birational to $\mathbb{P}^{n-1}$ over $k$.

Assume next that we have two points $p, q \in X$ and $C_{p}, C_{q}$ are both irreducible with a double point at $p$ (resp. $q$ ). Define the "3rd intersection point" map

$$
\phi: C_{p} \times C_{q} \rightarrow X
$$

as follows. Take $u \in C_{p}, v \in C_{q}$. If the line connecting $u, v$ is not contained in $X$, it has a unique 3rd intersection point with $X$; call it $\phi(u, v)$. Under very mild genericity assumptions (15) this is a well 
defined dominant map. Thus we get that $X$ is unirational via

$$
\Phi: \mathbb{P}^{n-1} \times \mathbb{P}^{n-1} \stackrel{\substack{\pi_{p} \times \pi_{q} \\-\rightarrow}}{\rightarrow} C_{p} \times C_{q} \stackrel{\phi}{-\rightarrow} X .
$$

Definition 7 (Restriction of scalars).

Let $L / K$ be a finite degree field extension. Restriction of scalars (or Weil restriction) is way to associate to an $L$-variety $U$ a $K$-variety $\mathfrak{R}_{L / K} U$ such that there is a natural identification of the $L$-points of $U$ with the $K$-points of $\mathfrak{R}_{L / K} U$. This dictates that $\operatorname{dim} \mathfrak{R}_{L / K} U=$ $\operatorname{deg}(L / K) \cdot \operatorname{dim} U$; see BLR90, 7.6] for details.

This can be done very explicitly in the affine case as follows. Let $U \subset$ $\mathbb{A}^{n}$ be an affine variety defined over $L$. Choose equations $f_{i}\left(x_{1}, \ldots, x_{n}\right)$ for $U$ and let $e_{1}, \ldots, e_{d} \in L$ be a $K$-basis. Choose new coordinates $y_{i j}: i=1, \ldots, n, j=1, \ldots, d$ and set $x_{i}=\sum_{j} e_{j} y_{i j}$. We can then write

$$
f_{k}\left(x_{1}, \ldots, x_{n}\right)=\sum_{\ell} e_{\ell} f_{k \ell}\left(y_{i j}\right) \quad \text { where } f_{k \ell} \in K\left[y_{i j}\right] \text {. }
$$

Let $\mathfrak{R}_{L / K} U$ be the subvariety of $\mathbb{A}^{n d}$ defined by the equations $f_{k \ell}=0$.

In particular we see that $\mathfrak{R}_{L / K}\left(\mathbb{A}^{n}\right) \cong \mathbb{A}^{d n}$. In the projective case, $\mathfrak{R}_{L / K}\left(\mathbb{P}^{n}\right)$ is inconvenient to describe by explicit equations but we at least get that $\mathfrak{R}_{L / K}\left(\mathbb{P}^{n}\right)$ is birational to $\mathbb{P}^{d n}$ over $K$. (They are not isomorphic for $d>1$.)

8 (Second unirationality construction).

Let $X \subset \mathbb{P}^{n+1}$ be a cubic defined over $k$ and $k^{\prime} \supset k$ a quadratic extension. Let $p \in X\left(k^{\prime}\right)$ be a point and $\bar{p} \in X\left(k^{\prime}\right)$ its conjugate. (Let us ignore that $k^{\prime} \supset k$ may be inseparable in characteristic 2.) We have conjugate birational maps $\pi_{p}: \mathbb{P}^{n-1} \rightarrow C_{p}$ and $\pi_{\bar{p}}: \mathbb{P}^{n-1} \rightarrow C_{\bar{p}}$. If $u \in \mathbb{P}^{n-1}\left(k^{\prime}\right)$ then $\pi_{p}(u)$ and $\pi_{\bar{p}}(\bar{u})$ are conjugate points of $X$, thus the line connecting them is defined over $k$. Hence $\phi(u, \bar{u}) \in X(k)$. Putting this invariantly, we obtain a rational map (defined over $k$ )

$$
\Phi: \mathfrak{R}_{k^{\prime} / k} \mathbb{P}^{n-1} \rightarrow X
$$

which is dominant under mild genericity assumptions.

9 (Final unirationality construction).

Assume now that $X$ is a cubic defined over $k$ and $x \in X$ is a smooth $k$-point. Let $L$ be a line through $x$. If $L$ is not contained in $X$ then it intersects $X$ in a point pair $\{p, q\}$. These points are usually not in $k$, but they are conjugate over $k$ and lie in a quadratic extension $k^{\prime}=k^{\prime}(L)$ of $k$. Hence, under some genericity assumptions, we obtain a dominant map

$$
\Phi: \mathfrak{R}_{k^{\prime} / k} \mathbb{P}^{n-1} \rightarrow X
$$


which shows that $X$ is unirational. There are very few problems if $k$ is infinite, since then a general choice of $L$ should work. (Extra work is needed in characteristic 2.) The situation is less clear over finite fields since there may not be enough room to choose $L$ general; see, for example, (16).

To avoid this difficulty, we do not choose any line, rather we work with all lines simultaneously. We should obtain a map

$$
\Psi: \cup_{x \in L \subset \mathbb{P}^{n+1}} \mathfrak{R}_{k^{\prime}(L) / k} \mathbb{P}^{n-1} \rightarrow X .
$$

We are in good shape if we can indentify the left hand side with a product $\mathbb{P}^{n} \times \mathbb{P}^{n-1} \times \mathbb{P}^{n-1}$, at least birationally. Once this problem is settled, it is enough to check dominance over the algebraic closure where the previous arguments work. It seems best to give an explicit algebraic description.

10 (Algebraic description of $\Psi$ ).

We work in affine coordinates, assuming that the origin is a smooth point of $X$. Thus the equation of $X$ can be written as

$$
F=L\left(x_{1}, \ldots, x_{n+1}\right)+Q\left(x_{1}, \ldots, x_{n+1}\right)+C\left(x_{1}, \ldots, x_{n+1}\right)
$$

where $L$ is linear, $Q$ is quadratic and $C$ is cubic. We may assume that $\partial F / \partial x_{n+1}$ is not indetically zero (for instance we can even assume that $\left.L=x_{n+1}\right)$.

We write down a rational map

$$
\Psi: \mathbb{A}^{3 n-2}\left(u_{1}, \ldots, u_{n}, v_{1}, \ldots, v_{n-1}, w_{1}, \ldots, w_{n-1}\right) \rightarrow X .
$$

Later we check that it is dominant with a few exceptions.

Consider the universal line through the origin $\left(\tau u_{1}, \ldots, \tau u_{n}, \tau\right)$. It intersects $X$ in two further points which correspond to the roots of the quadratic equation

$$
L\left(u_{1}, \ldots, u_{n}, 1\right)+\tau Q\left(u_{1}, \ldots, u_{n}, 1\right)+\tau^{2} C\left(u_{1}, \ldots, u_{n}, 1\right)=0 .
$$

The equation is irreducible if $X$ is irreducible. Let its roots be $t_{1}, t_{2} \in$ $\overline{k\left(u_{1}, \ldots, u_{n}\right)}$.

The equation of the tangent space of $X$ at $\mathbf{p}=\left(p_{1}, \ldots, p_{n+1}\right) \in X$ is

$$
\frac{\partial F}{\partial x_{1}}(\mathbf{p})\left(x_{1}-p_{1}\right)+\cdots+\frac{\partial F}{\partial x_{n+1}}(\mathbf{p})\left(x_{n+1}-p_{n+1}\right)=0 .
$$

Thus the universal tangent line at $\left(t_{1} u_{1}, \ldots, t_{1} u_{n}, t_{1}\right)$ can be described parametrically as

$$
\begin{aligned}
& x_{1}=t_{1} u_{1}+\sigma\left(v_{1}+t_{1} w_{1}\right), \ldots, x_{n-1}=t_{1} u_{n-1}+\sigma\left(v_{n-1}+t_{1} w_{n-1}\right) \\
& x_{n}=t_{1} u_{n}+\sigma \\
& x_{n+1}=t_{1}-\sigma\left(\frac{\partial F}{\partial x_{n+1}}\left(t_{1} \mathbf{u}, t_{1}\right)\right)^{-1} \sum_{i=1}^{n} \frac{\partial F}{\partial x_{i}}\left(t_{1} \mathbf{u}, t_{1}\right)\left(v_{i}+t_{1} w_{i}\right),
\end{aligned}
$$


where we set $v_{n}=1, w_{n}=0$. Substituting the above parametric representation into $F$, we obtain a cubic equation in $\sigma$

$$
\sum_{j=0}^{3} \sigma^{j} H_{j} \quad \text { where } \quad H_{j} \in k\left(\mathbf{u}, \mathbf{v}, \mathbf{w}, t_{1}\right) .
$$

$H_{0}=H_{1}=0$ since we have a tangent line, thus the 3rd intersection point corresponds to the value $\sigma=-H_{2} / H_{3}$. Thus we obtain a point

$$
Q_{1} \in k\left(\mathbf{u}, \mathbf{v}, \mathbf{w}, t_{1}\right)^{n+1} .
$$

Replacing $t_{1}$ by its conjugate $t_{2}$ we obtain another point $Q_{2}$. The line connecting $Q_{1}$ and $Q_{2}$ can be given parametrically as

$$
L(\lambda)=\frac{\lambda-t_{2}}{t_{1}-t_{2}} Q_{1}+\frac{\lambda-t_{1}}{t_{2}-t_{1}} Q_{2}
$$

and this is a parametrization over $k(\mathbf{u}, \mathbf{v}, \mathbf{w})$. Evaluating $F$ on the line we have that $t_{1}, t_{2}$ are roots, so

$$
F(L(\lambda))=(A \lambda+B)\left(C \lambda^{2}+Q \lambda+L\right) .
$$

Thus if we expand

$$
F(L(\lambda))=\sum_{j=0}^{3} \lambda^{j} G_{j}, \quad \text { then } \quad G_{j} \in k(\mathbf{u}, \mathbf{v}, \mathbf{w}),
$$

and the 3rd root is

$$
-\frac{B}{A}=-\frac{G_{2}}{G_{3}}+\frac{Q}{C}
$$

Substituting this into the parametrization of the line gives

$$
\Psi(\mathbf{u}, \mathbf{v}, \mathbf{w}) \in X(k(\mathbf{u}, \mathbf{v}, \mathbf{w})) .
$$

Depending on our definition of unirationality, we also need to check the following:

Lemma 11. For a $k$-variety $X$ the following are equivalent:

1. There is a dominant map $\phi_{m}: \mathbb{A}^{m} \rightarrow X$ for some $m$.

2. There is a dominant map $\phi_{m}: \mathbb{A}^{m} \rightarrow X$ for $m=\operatorname{dim} X$.

Proof. Assume that $m>\operatorname{dim} X$. There is a dense open set $U \subset \mathbb{A}^{m}$ such that $\left.\phi_{m}\right|_{U}$ is open with $m-\operatorname{dim} X$ dimensional fibers. Let $\mathbf{u} \in U$ be a point. If $\mathbf{u} \in Z \subset \mathbb{A}^{m}$ is a hypersurface which does not contain the irreducible component of the fiber of $\left.\phi_{m}\right|_{U}$ through $\mathbf{u}$, then $\left.\phi_{m}\right|_{Z}$ : $Z \rightarrow X$ is dominant.

If $k$ is infinite and $m>\operatorname{dim} X$ then we can choose $Z$ to be a general hyperplane.

Assume next that $k$ is finite. Fix a prime $\ell \neq$ char $k$ and let $k^{\prime}$ be the composite of all algebraic extensions of degree $\ell^{s}$ of $k . k^{\prime}$ is infinite, 
hence we can choose a point $\mathbf{u}=\left(u_{1}, \ldots, u_{m}\right) \in U\left(k^{\prime}\right)$. By permuting the coordinates we may assume that $\operatorname{deg} k\left(u_{m}\right) / k \leq \operatorname{deg} k\left(u_{1}\right) / k$, or, equivalently, $k\left(u_{m}\right) \subset k\left(u_{1}\right)$. This implies that $u_{m}$ can be written as a polynomial of $u_{1}$, hence the ideal $I(\mathbf{u}) \subset k\left[x_{1}, \ldots, x_{m}\right]$ contains an polynomial of the form $x_{m}-p\left(x_{1}\right)$. This implies that $I(\mathbf{u})$ is generated by polynomials of the form $x_{m}-P\left(x_{1}, \ldots, x_{m-1}\right)$. Thus we can choose $Z=\left(x_{m}=P\left(x_{1}, \ldots, x_{m-1}\right)\right)$ for suitable $P$.

12 (Proof of (2.3) $\Rightarrow(2 \cdot 1)$ ).

Let $X$ be an irreducible cubic hypersurface. The set of all triple points of $X(\bar{k})$ is a linear space and it is defined over $k$ if $k$ is perfect. Thus $X$ is a cone over a cubic hypersurface without triple points. Therefore, $X$ has no triple points over $\bar{k}$.

Assume next that $X$ is not normal. The nonnormal locus has dimension $(n-1)$ and the linear space spanned by it is in $X$. Thus the nonnormal locus is a linear space $L^{n-1} \subset \mathbb{P}^{n+1}$ which is defined over $k$ if $k$ is perfect. Projecting form $L$ realizes $X$ as a $\mathbb{P}^{n-1}$-bundle over $\mathbb{P}^{1}$, hence rational.

For the rest of the proof assume that $X$ is normal. We need to check three conditions.

First we prove that $C_{x}$ is irreducible with a double point at $x$ for general $x \in X(\bar{k})$. This is done in (14).

Second, we need to check that the 3rd intersection point map $\phi$ : $C_{p} \times C_{q} \rightarrow X$ is dominant. It is, however, not enough to check this for a general pair $p, q$. In our construction $p, q$ are the two intersection points of a line through $x$, hence dependent. Assume that $\pi_{x}: X \rightarrow \mathbb{P}^{n}$, the projection from $x$, is separable. Then for a generic line $x \in L$ we get 2 distinct intersection points and both intersections are transverse. In particular, the tangent space of $X$ at one point does not contain the other point. In (15) we see that this is sufficient to guarantee that $\phi: C_{p} \times C_{q} \rightarrow X$ is dominant.

Third, we need to consider the case when the projection $\pi_{x}: X \rightarrow-$ $\mathbb{P}^{n}$ is inseparable. This can happen only in characteristic 2. Over a perfect field a purely inseparable map induces a purely inseparable map in the reverse direction, hence in this case $X$ is (purely inseparably) unirational. Nonetheless, we check in (20) that we can always choose a smooth $k$-point such that projection from it is separable.

By looking at the proof we obtain the following for nonperfect fields. We check in (18) that (13.3) is satisfied for $X$ smooth. This shows that our proof covers all smooth hypersurfaces. 
Proposition 13. Let $k$ be a nonperfect field and $X \subset \mathbb{P}^{n+1}$ a cubic hypersurface which is not a cone. Then the 3 parts of (2) are equivalent if one of the following conditions holds:

1. char $k \geq 5$.

2. char $k=3$ and $X$ has no triple points over $\bar{k}$.

3. char $k=2$ and there is a smooth $k$ point $p \in X$ such that projection from $p$ is separable.

Proposition 14. Let $k$ be an algebraically closed field and $X \subset \mathbb{P}^{n+1}$ a normal cubic hypersurface over $k$ without triple points. Then $C_{x}$ is irreducible with a double point at $x$ for general $x \in X$.

Proof. Let $x \in X$ be arbitrary. If $C_{x}$ is irreducible with a triple point at $x$ then $C_{x}$ is a cone, hence there is an $(n-2)$-dimensional family of lines through $x$. If $C_{x}$ is reducible then either $C_{x}$ contains an $(n-1)$-dimensional linear space through $x$ or a quadric cone with vertex at $x$. In either case, there is an $(n-2)$-dimensional family of lines through $x$. Thus it is enough to prove that for a general $x \in X$ the family of lines in $X$ through $x$ has dimension at most $n-3$. This is equivalent to proving that a general surface section through $x$ has no lines through $x$.

$X$ has no triple points, hence by Bertini, a general surface section of $X$ is also normal with no triple points.

If $S$ is a normal cubic surface without triple points then there are only finitely many lines through each double point. (Choose affine coordinates such that the equation becomes $q\left(x_{1}, x_{2}, x_{3}\right)+c\left(x_{1}, x_{2}, x_{3}\right)=0$. The lines through $(0,0,0)$ correspond to the solutions of $(q=c=0) \subset$ $\mathbb{P}^{2}$. If there are infinitely many solutions, then $q$ and $c$ have a common factor, thus the surface is reducible.) Each line in the smooth locus has selfintersection -1 , hence rigid. Thus $S$ has only finitely many lines.

Lemma 15. Let $X \subset \mathbb{P}^{n+1}$ be an irreducible cubic hypersurface. Let $x, y \in X$ be smooth points and $C_{x}, C_{y}$ the corresponding intersections with the tangent hyperplanes. Assume that

1. $C_{x}$ and $C_{y}$ are irreducible.

2. $x \notin C_{y}$ and $y \notin C_{x}$.

Then the 3rd intersection point map $\phi: C_{x} \times C_{y} \rightarrow X$ is dominant.

Proof. Let us see first that $\phi$ is indeed defined. Pick a point $u \in C_{x}$ which is a smooth point of $X$. Pick $v \in C_{y}$ which is a smooth point of $X$ such that $v$ does not lie on $T_{u} X$. If we now choose a general $w \in C_{x}$ then $v$ does not lie on $T_{w} X$ and $w$ does not lie on $T_{v} X$. Thus 
the line connecting $u, w$ has a unique third intersection point with $X$. This shows that $\phi$ is defined at the pair $(v, w)$.

In order to prove dominance, we need to show that $\phi$ has at least one fiber of dimension $n-2$. Pick a point $z \in X$ which is not on $C_{x} \cup C_{y}$ and let $\pi: \mathbb{P}^{n+1} \rightarrow T_{y} X$ denote the projection from $z$. Then $\phi^{-1}(z)$ is the set of pairs $(v, w)$ such that $\pi(v)=w$. Thus we are done if

$$
\operatorname{dim}\left(\left(C_{y} \cap \pi\left(C_{x}\right)\right) \backslash\left(C_{y} \cap C_{x}\right)\right)=n-2 .
$$

For this it is sufficient to find one projection $\pi^{\prime}: \mathbb{P}^{n+1} \rightarrow T_{y} X$ where this holds. Then the same holds for a general projection and a general projection always corresponds to a point of $X$. Pick any smooth point $v \in C_{y}$ and let $\pi^{\prime}$ be a projection such that $\pi^{\prime}(x)=v$. Then $\pi^{\prime}\left(C_{x}\right)$ and $C_{y}$ intersect at $v$ but they have different multiplicty there. Hence their intersection has dimension $n-2$.

Example 16. Let $S$ be the cubic surface $\left(x_{0}^{3}+x_{1}^{3}+x_{2}^{3}+x_{3}^{3}=0\right)$. By Hirschfeld81] over the fields $\mathbb{F}_{2}, \mathbb{F}_{4}, \mathbb{F}_{16}$ all the points are on the 27 lines. Hence the second unirationality construction does not work over $\mathbb{F}_{2}$ and $\mathbb{F}_{4}$. (It does work over $\mathbb{F}_{16}$.)

Example 17. Let $k$ be a field of characteristic 3 and $t_{i}$ algebraically independent over $k$. Set $K=k\left(t_{1}, \ldots, t_{n}\right)$ and

$$
Y:=\left(y^{3}-y z^{2}=\sum_{i=1}^{n} t_{i} x_{i}^{3}\right) \subset \mathbb{P}^{n+1} .
$$

1. $Y$ is non-singular.

2. Over $\bar{K}, Y$ is a cone over a cuspidal cubic curve.

3. $Y(K)=\{(0,1,0, \ldots, 0),(1,1,0, \ldots, 0),(1,-1,0, \ldots, 0)\}$.

4. $Y$ is not unirational (over $K$ ).

Proof. $Y$ is the generic fiber of the smooth variety

$$
\left(y^{3}-y z^{2}=\sum_{i} t_{i} x_{i}^{3}\right) \subset \mathbb{A}_{\left(t_{1}, \ldots, t_{n}\right)}^{n} \times \mathbb{P}_{\left(y, z, x_{1}, \ldots, x_{n}\right)}^{n+1}
$$

over $\mathbb{A}^{n}$, thus $Y$ is non-singular. (2) holds since over $\bar{K}$ we can write our equation as

$$
\left(y-\sum_{i} \sqrt[3]{t_{i}} x_{i}\right)^{3}-y z^{2}=0 .
$$

In order to see (3) we may as well assume that $k$ is algebraically closed. Assume that we have relatively prime polynomials $f, g, h_{i} \in$ $k\left[t_{1}, \ldots, t_{n}\right]$ such that

$$
f^{3}-f g^{2}=\sum_{i} t_{i} h_{i}^{3}
$$


We are done if $h_{1}=\cdots=h_{n}=0$. Otherwise, we can make a substitution $t_{i}=c_{i} t$ for $i=1, \ldots, n$ and general $c_{i}$ to get a solution of

$$
f(f-g)(f+g)=t \cdot h^{3} \quad \text { with } f, g, h \in k[t] \text { and } h \neq 0 .
$$

We may assume that $f$ and $g$ are relatively prime. Thus 2 of the factors $f, f-g, f+g$ are cubes and the third is $t$ times a cube. However, $f+(f-g)+(f+g)=0$, hence if 2 are cubes then so is their sum which is minus the 3rd factor. This is a contradiction.

Since $Y$ has only 3 points in $K$, it does not contain any rational curves and so it is definitely not unirational.

Lemma 18. Assume that char $k=2$. Let $V \subset \mathbb{P}^{n+1}$ be the linear span of all points $p \in X(k)$ such that projection from $p$ is a purely inseparable map $X \rightarrow \mathbb{P}^{n}$. Let $\left(y_{i}=0\right)$ be equations of $V$ and $x_{j}$ coordinates on $V$. Then the equation of $X$ can be written as

$$
f:=\sum_{j} \ell_{j}(\mathbf{y}) x_{j}^{2}+g(\mathbf{y})
$$

where the $\ell_{j}$ are linear and $g$ is cubic. If $V \neq \emptyset$ then $X$ is not smooth.

Proof. We can choose coordinates such that the points

$$
p_{1}=(1: 0: \cdots: 0), \ldots, p_{m}=\left(0: \cdots::^{m-t h}: 0: \cdots: 0\right)
$$

are in $X(k)$ and projection from $p_{i}$ is a purely inseparable map for $i=1, \ldots, m \cdot p_{i}$ is inseparable iff $x_{i}$ occurs in the equation of $X$ always with even exponent. This gives the above equation.

$\partial f / \partial x_{j}$ is zero, and the equations $\partial f / \partial y_{i}=0$ have a common solution. Since $f=3 f=\sum_{i}\left(\partial f / \partial y_{i}\right)$, these give singular points of $X(\bar{k})$.

Lemma 19. Let $k$ be a perfect field of characteristic 2. Let $X$ be a cubic of dimension at least 2 given by an equation

$$
f(\mathbf{x}, \mathbf{y}):=\sum_{j} \ell_{j}(\mathbf{y}) x_{j}^{2}+g(\mathbf{y}) .
$$

Then $X$ has a smooth $k$-point with nonzero $y$-coordinate.

Proof. Assume first that we have at least two $x$-variables. If $\ell_{1}=c \ell_{2}$ then

$$
\ell_{1} x_{1}^{2}+\ell_{2} x_{2}^{2}=\ell_{1}\left(x_{1}+\sqrt{c} x_{2}\right)^{2}
$$

thus we can change coordinates to eliminate one $x$-variable. Otherwise we can pick $\mathbf{y}_{0}$ such that $\ell_{1}\left(\mathbf{y}_{0}\right) \neq 0$ and $\ell_{2}\left(\mathbf{y}_{0}\right)=0$. Then

$$
\left(\sqrt{g\left(\mathbf{y}_{0}\right) / \ell_{1}\left(\mathbf{y}_{0}\right)}, x_{2}, \mathbf{y}_{0}\right) \in X(k)
$$


for every $x_{2}$. Since

$$
\frac{\partial f}{\partial y_{i}}=x_{2}^{2}+\frac{\partial g}{\partial y_{i}}
$$

the above point is smooth for suitable choice of $x_{2}$.

Thus assume that there is only one $x$-variable and write the equation as $y_{1} x_{1}^{2}+g(\mathbf{y})$.

Take any $\left(p_{1}, \ldots, p_{n}\right) \in k^{n}$. If $p_{1}=0$ and $g\left(p_{1}, \ldots, p_{n}\right)=0$ then $\left(x_{1}: p_{1}: \cdots: p_{n}\right) \in X(k)$ for any $x_{1}$ and one of them is a smooth by looking at $\partial f / \partial y_{1}$.

If $p_{1} \neq 0$ then $p_{0}:=\sqrt{-g\left(p_{1}, \ldots, p_{n}\right) / p_{1}} \in k$ and $\left(p_{0}: p_{1}: \cdots: p_{n}\right)$ is a smooth point unless

$$
g\left(p_{1}, \ldots, p_{n}\right)-p_{1} \frac{\partial g}{\partial y_{1}}\left(p_{1}, \ldots, p_{n}\right)=0 .
$$

Thus we are done unless the following holds:

1. $g-y_{1}\left(\partial g / \partial y_{1}\right)$ is nonzero for $y_{1}=0$, and

2. $g-y_{1}\left(\partial g / \partial y_{1}\right)$ is zero for $y_{1} \neq 0$.

Write $g=\sum y_{1}^{i} g_{3-i}\left(y_{2}, \ldots, y_{n}\right)$. Then

$$
g-y_{1}\left(\partial g / \partial y_{1}\right)=y_{1}^{2} g_{1}\left(y_{2}, \ldots, y_{n}\right)+g_{3}\left(y_{2}, \ldots, y_{n}\right)
$$

$g_{1}$ is a linear form thus it has a nontrivial zero $\left(p_{2}, \ldots, p_{n}\right)$. If $g_{3}\left(p_{2}, \ldots, p_{n}\right)=$ 0 then set $p_{1}=0$ and if $g_{3}\left(p_{2}, \ldots, p_{n}\right) \neq 0$ then set $p_{1}=1$.

Combining the above lemmas we obtain:

Corollary 20. Let $k$ be a perfect field of characteristic 2 and $X \subset \mathbb{P}^{n+1}$ a cubic with a smooth $k$-point. Assume that $n \geq 2$. Then there is a smooth point $x \in X(k)$ such that the projection from $x$ is separable.

Acknowledgments . I thank J.-L. Colliot-Thélène and J. Ellenberg for helpful comments and references. Partial financial support was provided by the NSF under grant number DMS-9970855.

\section{REFERENCES}

BLR90. S. Bosch, W. Lütkebohmert and M. Raynaud, Néron models, Ergebnisse der Mathematik und ihrer Grenzgebiete, Springer-Verlag, Berlin, 1990

CT-S-SD87. J.-L. Colliot-Thélène, J.-J. Sansuc and P. Swinnerton-Dyer, Intersections of two quadrics and Châtelet surfaces I, J. f.r.u.a. Math. 373 (1987) 37-107

Hirschfeld81. J. Hirschfeld, Cubic surfaces whose points all lie on their 27 lines, in: Finite geometries and designs (Proc. Conf. Chelwood Gate, 1980) 169-171, London Math. Soc. Lecture Note vol. 49, Cambridge Univ. Press, Cambridge-New York, 1981 
Manin72. Yu. I. Manin, Cubic forms (in Russian) Nauka, 1972; English translation: North-Holland 1974, second enlarged edition, 1986

Nishimura55. H. Nishimura, Some remarks on rational points, Mem. Coll. Sci. Univ. Kyoto, 29 (1955) 189-192

Segre43. B. Segre, A note on arithmetical properties of cubic surfaces, J. London Math. Soc. 18 (1943) 24-31

Princeton University, Princeton NJ 08544-1000

kollar@math.princeton .edu 